This is the author's copy of the publication as archived with the DLR's electronic library at http://elib.dlr.de . Please consult the original publication for citation, see e.g.

\title{
Adaptive Washout Filter Based on Fuzzy Logic for a Motion Simulation Platform With Consideration of Joints' Limitations
}


Saeid Nahavandi

Motion simulation platforms (MSPs) are widely used to generate driving/flying motion sensations for the users. The MSPs have a restricted workspace area due to the dynamical and physical restrictions of the Motion Platforms active joints as well as the physical limitations of its passive joints. The motion cueing algorithm (MCA) is the reproduction of the motion signal including linear accelerations and angular velocities. It aims to simultaneously respect the MSP's workspace limitations and make the same motion feeling for the user as a real vehicle. The Classical washout filter (WF) is a wellknown type of MCA. The classical WF is easy to set-up, offers a low computational burden and high functionality but has some major drawbacks such as fixed WF parameters tuned according to worstcase scenarios and no consideration of the human vestibular system. As a result, adaptive WFs were developed to consider the human vestibular system and enhance the efficiency of the method using time-varying filters. The existing adaptive WFs only cogitate the boundaries of the end-effector in the Cartesian coordinate space as a substitute for the active and passive joints limitations, which is MSP's main limiting factor. This conservative assumption reduces the available workspace area of the MSP and increases the motion sensation error for the MSPs user. In this study, a fuzzy logic-based WF is developed, to consider the dynamical and physical boundaries of the active joints as well as the physical boundaries of the passive joints. A genetic algorithm is used to select the membership functions' values of the active and passive joints' boundaries. The model is designed using MATLAB /Simulink and the outcomes demonstrate the efficiency of the proposed method versus existing adaptive WFs.

\section{Copyright Notice}

(C2020 IEEE. Personal use of this material is permitted. Permission from IEEE must be obtained for all other uses, in any current or future media, including reprinting/republishing this material for advertising or promotional purposes, creating new collective works, for resale or redistribution to servers or lists, or reuse of any copyrighted component of this work in other works.

M. R. Chalak Qazani, H. Asadi, T. Bellmann, S. Mohamed, C. P. Lim and S. Nahavandi, "Adaptive Washout Filter Based on Fuzzy Logic for a Motion Simulation Platform With Consideration of Joints' Limitations," in IEEE Transactions on Vehicular Technology, vol. 69, no. 11, pp. 12547 12558, Nov. 2020, doi: 10.1109/TVT.2020.3023478. 


\title{
Adaptive Washout Filter Based on Fuzzy Logic for a Motion Simulation Platform with Consideration of Joints' Limitations
}

\author{
Mohammad Reza Chalak Qazani ${ }^{1}$, Houshyar Asadi ${ }^{2}$, Member, IEEE, Tobias Bellmann ${ }^{3}$, Shady \\ Mohamed $^{4}$, Chee Peng Lim ${ }^{5}$, Saeid Nahavandi ${ }^{6}$, Fellow, IEEE
}

\begin{abstract}
Motion simulation platforms (MSPs) are widely used to generate driving/flying motion sensations for the users. The MSPs have a restricted workspace area due to the dynamical and physical restrictions of the Motion Platforms active joints as well as the physical limitations of its passive joints. The motion cueing algorithm (MCA) is the reproduction of the motion signal including linear accelerations and angular velocities. It aims to simultaneously respect the MSP's workspace limitations and make the same motion feeling for the user as a real vehicle. The Classical washout filter (WF) is a well-known type of MCA. The classical WF is easy to set-up, offers a low computational burden and high functionality but has some major drawbacks such as fixed WF parameters tuned according to worst-case scenarios and no consideration of the human vestibular system. As a result, adaptive WFs were developed to consider the human vestibular system and enhance the efficiency of the method using timevarying filters. The existing adaptive WFs only cogitate the boundaries of the end-effector in the Cartesian coordinate space as a substitute for the active and passive joints limitations, which is MSP's main limiting factor. This conservative assumption reduces the available workspace area of the MSP and increases the motion sensation error for the MSPs user. In this study, a fuzzy logic-based WF is developed, to consider the dynamical and physical boundaries of the active joints as well as the physical boundaries of the passive joints. A genetic algorithm is used to select the membership functions' values of the active and passive joints' boundaries. The model is designed using MATLAB /Simulink and the outcomes demonstrate the efficiency of the proposed method versus existing adaptive WFs.
\end{abstract}

Index Terms - motion cueing algorithm, fuzzy logic, dynamical and physical limitations, hexapod platform, human sensation error, washout filter (WF), genetic algorithm.

\section{INTRODUCTION}

$\mathrm{T}$ HE reproduction of vehicle motion signals along 6-degrees of freedom (6-DoF) has become possible due to the use of Hexapod manipulators, known as one of the most commonly known parallel mechanism. The usage of parallel and serial motion simulation platforms (MSPs) has been growing recently in many industries including aviation, automotive and transportation [1,2]. The hexapod based MSPs have a limited workspace area due to the dynamical and physical limitations

(Corresponding author: Mohammad Reza Chalak Qazani)

1,2,4,5,6 The authors are with the Institute for Intelligent Systems Research and Innovation, Deakin University, Geelong, VIC 3125, Australia (e-mail: m.r.chalakqazani@gmial.com; of the active joints as well as the physical boundaries of the passive joints [3-6]. A motion cueing algorithm (MCA) is the main algorithm in charge of producing real vehicle motion cues within the dynamical and physical limitations of the MSPs, aiming to produce realistic driving/flying motion sensations for the MSP's user[7].

There are four different types of MCAs known as classical, adaptive, optimal [8-10] and model predictive control-based MCAs [11, 12]. Conrad and Schmidt [13] conducted a study which lead to developing classical washout filters (WFs) as the most common and commercial type of MCAs. It consists of a series of high- and low-pass filters with the addition of a tilt coordination channel which is responsible for the generation of sustainable accelerations from the low-frequency part of the motion signal via somatogravic illusion [14]. On the other hand, the high-pass filter employs high-frequency parts of the vehicle motion signals to move the MSP within its workspace. Cases et al. [15] used a genetic algorithm to find the optimal indexes of the classical MCAs without practical evaluation. The advantages of the classical WF include simplicity, high functionality and low computational load. Classical WFs should be tunned based on the worst-case scenario to restrict the end-effector inside the workspace in any circumstances. This assumption reduces the efficiency of the algorithm in reproducing accurate motion sensation signals. Also, classical WFs suffer from other drawbacks including fixed WF parameters, absence of a human vestibular model and lack of consideration for MSP's limitations. These drawbacks lead to motion sickness as the most important drawback of the MCA [16]. The concept of the adaptive WF was introduced by Parrish et al. [17] to change cut-off frequencies of the classical WF. Reid and Nahon [18, 19] employed the coordinated adaptive WF aiming to minimise motion sensation errors between the real vehicle driver and the MSP driver. The adaptive WF for 2DoF MSPs using gradient descent optimisation to extract the optimal coefficient parameters of the filters based on the motion sensation errors and the position and velocity limitations of the MSP was developed by Nehaoua et al. [20]. Zadeh [21] introduced the fuzzy logic theory that has been developed in Zentrum für Luft- und Raumfahrt (DLR), German Aerospace Center, 82234 Oberpfaffenhofen, Germany (e-mail: Tobias.Bellmann@dlr.de). 
control concepts. Fuzzy logic is one of the best techniques for systems with stochastic behaviour and changeable inputs because it is able to transform deterministic plans to stochastic plans [22, 23]. Hwang et al. [24] employed fuzzy logic to change the fixed parameters of WFs for the generation of accurate motion signals. Asadi et al. $[25,26]$ employed the fuzzy logic controller to fluctuate the cut-off frequencies of the classical WF based on the motion sensation error between the real vehicle driver and the MSP driver, and the translational and rotational displacement of the MSP. The consideration for the MSP's limitation $[25,26]$ in the Cartesian space for serial MSPs works, however, it reduces the efficiency of the presented algorithm for Hexapod MSPs. The primary reasons for the workspace limitation are the dynamical and physical boundaries of the active joints as well as the physical limitations of the passive joints. Another aspect of the adaptive WF is introduced by Asadi et al. [27-29] by generating motion compensation signals based on the contemporary situation of the MSPs such as end-effector distance from the neutral position.

A mid-sized Hexapod MSP, which is shown in Fig. 1, is employed in this study since it this type parallel manipulators are the most common parallel manipulators for the generation of motions in 6-DoF [30]. The workspace area, dynamical and physical boundaries of the active joints and the physical boundaries of the passive joints are shown in Table I.

All previous studies on classical and adaptive WFs [13, 17$20,24-28,31]$ generate the motion signal based on the MSP's boundaries in the Cartesian space. Also, they did not consider the dynamical boundaries of the active joints including its acceleration and velocity limitations. The consideration of the dynamical and physical boundaries of the active joints leads to efficient usage of the platform workspace, enhances the motion fidelity and causes better generation of the high-frequency motion signals. The physical boundaries of the passive joints are considered in this study to use the maximum angular displacement of the MSP based on the boundaries of the passive joints' angles. A fuzzy logic controller is employed in this research to formulate the new adaptive WF while considering the dynamical and physical limitations of the MSP's actuators as well as the physical boundaries of its passive joints. The main purpose in developing of the proposed fuzzy logic-based adaptive WF is to overcome the disadvantages of the existing adaptive WFs, including the lack of consideration of the joints' limitations [17, 27, 28, 32]. The proposed WF monitors the accelerations, velocities and positions of the hexapod's actuators, angular position of the passive joints and driving sensation error between the real vehicle driver and the MSP driver aiming to generate the appropriate motion signals. This is to solve the inefficient usage of the workspace area which constitutes an artefact for the driver of the MSP. The fuzzy logic controllers are employed in this study to extract the appropriate cut-off frequency for the high- and low-pass filters in the adaptive WF. Also, the membership functions of the proposed fuzzy logic controllers are selected using a genetic algorithm aiming to minimise the motion sensation error.

The inverse kinematics model of the Hexapod MSP is
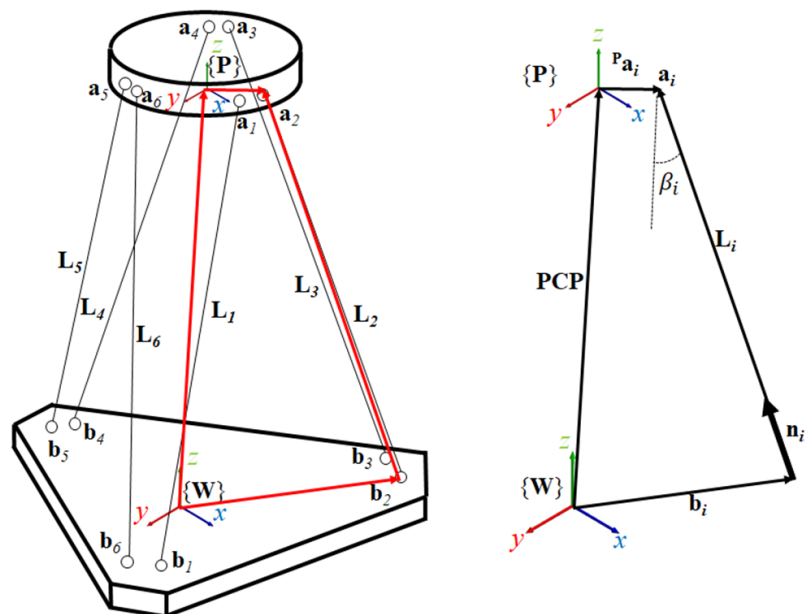

Fig. 1. The structure and the closed kinematic chain of the Hexapod MSP.

explained in section II. The presented fuzzy logic-based adaptive WF is explained in section III. Also, the outcomes of the motion scenario are disputed in section III. The concluding comments are disputed in section IV.

\section{INVERSE KinEMATIC MODEl OF THE HeXAPOD MSP}

The inverse kinematic model of the Hexapod MSP is in charge of calculating the accelerations, velocities and positions of the actuators based on the acceleration, velocity and position of the end-effector [33, 34]. Fig. 1 shows the 3D file and the structure of the Hexapod MSP. According to the closed kinematic chain of the Hexapod MSP, the length vector $\mathbf{L}_{i}$ of the $i^{\text {th }}$ Hexapod leg results from the equation:

$\mathbf{L}_{i}+\mathbf{b}_{i}=\mathbf{P C P}+\mathbf{a}_{i}$

where $\mathbf{a}_{i}$ and $\mathbf{b}_{i}$ are the position vectors of the upper and lower passive joints of the Hexapod MSP which are attached to the fixed platform and moving platform, respectively. The endeffector centre point, shown as PCP, shows the position vector of the Hexapod MSP in the world frame $\{\mathbf{W}\} . \mathbf{a}_{i}$ can be calculated as:

$\mathbf{a}_{i}=\mathbf{R} \mathbf{P} \mathbf{a}_{i}$

where $\mathbf{R}$ is the rotation matrix along $X Y Z$ which indicates the rotation from a local frame $\{\mathbf{P}\}$ to the world frame $\{\mathbf{W}\} .{ }^{\mathrm{P}} \mathbf{a}_{i}$ is the fixed position vector of the passive upper joints on the moving platform with respect to the local frame $\{\mathbf{P}\}$.

If $\mathbf{n}_{i}$ is the unit vector of the Hexapod leg and the length of the $i^{\text {th }}$ leg is $l_{i}$, the length of the Hexapod leg can be extracted as follows:

$l_{i} \mathbf{n}_{i}=\mathbf{P C P}+\mathbf{R}^{\mathbf{P}} \mathbf{a}_{i}-\mathbf{b}_{i}$.

The speed of the Hexapod $i^{\text {th }}$ leg $i_{\imath}$ can be calculated by taking the derivative and dot product with $\mathbf{n}_{i}$ of the Eq. (4) as follows:

$\dot{l}_{\imath}=\left(\mathbf{P C} \mathbf{P}+\boldsymbol{\omega} \times \mathbf{R}^{\mathbf{P}} \mathbf{a}_{i}\right) \cdot \mathbf{n}_{i}$,

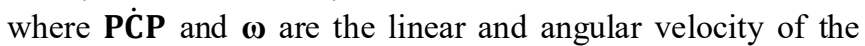
Hexapod MSP, respectively.

The acceleration of the $i^{\text {th }}$ leg, $\ddot{l}_{i}$, can be found by taking the derivative of Eq. (5) with respect to time as: 
TABLE I

THE DYNAMICAL AND PHYSICAL BOUNDARIES OF THE HEXAPOD MSP

\begin{tabular}{cccc}
\hline \hline Index & Position & Velocity & Acceleration \\
\hline$l_{i}$ & $0.76-1.13(\mathrm{~m})$ & $\pm 0.23(\mathrm{~m} / \mathrm{s})$ & $\pm 4\left(\mathrm{~m} / \mathrm{s}^{2}\right)$ \\
$\beta_{i}$ & $\pm 12(\mathrm{deg})$ & NA & NA \\
$x$ & $\pm 0.1(\mathrm{~m})$ & $\pm 1.5(\mathrm{~m} / \mathrm{s})$ & $\pm 3.3\left(\mathrm{~m} / \mathrm{s}^{2}\right)$ \\
$y$ & $\pm 0.1(\mathrm{~m})$ & $\pm 1.5(\mathrm{~m} / \mathrm{s})$ & $\pm 3.5\left(\mathrm{~m} / \mathrm{s}^{2}\right)$ \\
$Z$ & $\pm 0.02(\mathrm{~m})$ & $\pm 0.9(\mathrm{~m} / \mathrm{s})$ & $\pm 5.0\left(\mathrm{~m} / \mathrm{s}^{2}\right)$ \\
Roll & $\pm 10(\mathrm{deg})$ & $\pm 115(\mathrm{deg} / \mathrm{s})$ & $\pm 550\left(\mathrm{deg} / \mathrm{s}^{2}\right)$ \\
Pitch & $\pm 10(\mathrm{deg})$ & $\pm 55(\mathrm{deg} / \mathrm{s})$ & $\pm 550\left(\mathrm{deg} / \mathrm{s}^{2}\right)$ \\
Yaw & $\pm 12.5(\mathrm{deg})$ & $\pm 55(\mathrm{deg} / \mathrm{s})$ & $\pm 200\left(\mathrm{deg} / \mathrm{s}^{2}\right)$ \\
\hline \hline
\end{tabular}

deg: degree; m: meter; NA: not any; s: second.

$\ddot{l}_{i}=\left(\mathbf{P} \ddot{\mathbf{C}} \mathbf{P}+\boldsymbol{\alpha} \times \mathbf{R}^{\mathbf{P}} \mathbf{a}_{i}+\boldsymbol{\omega} \times\left(\boldsymbol{\omega} \times \mathbf{R}^{\mathbf{P}} \mathbf{a}_{i}\right)\right) \cdot \mathbf{n}_{i}$,

where $\mathbf{P C} \mathbf{P}$ and $\boldsymbol{\alpha}$ are the linear and angular acceleration of the Hexapod MSP, respectively.

There are two different types of passive joints in the structure of the Hexapod MSP. The universal passive joints connect the fixed plate of the Hexapod MSP to the legs, and the spherical passive joints attach the legs to the moving platform of the Hexapod MSP. The spherical joints typically have higher angular limitations compared with universal joints. Therefore, the passive upper joints should be extracted to be considered inside the adaptive WF. The angular displacement of the $i^{\text {th }}$ upper passive joints, $\beta_{i}$, can be found by taking the dot product between the normal vector of the legs' position and the normal vector of the Hexapod MSP's position as follows:

$\beta_{i}=\operatorname{acos}\left(\frac{\mathbf{n}_{i} \cdot \mathbf{n}_{P C P}}{\left|\mathbf{n}_{i}\right|\left|\mathbf{n}_{P C P}\right|}\right)$

where $\mathbf{n}_{P C P}$ is the unit vector of the Hexapod MSP's position.

The consideration of the legs' accelerations, velocities and positions as well as the angular displacements of the passive upper joints can enhance the ability of the proposed algorithm to generate more accurate driving signals based on the acceleration, velocity and position of the Hexapod MSP. The whole workspace area of the Hexapod MSP cannot be used efficiently due to the conservative presumption of the workspace limitations in the Cartesian space.

\section{FuZZY LOGIC-BASED AdAPTIVE WF}

The schematic structure of the proposed adaptive WF with consideration for the active and passive joints' limitations regarding a longitudinal channel is shown in Fig. 2. It consists of the second-order high- and low-pass filters in translational and tilt coordination channels, respectively. Also, a first-order high-pass filter is employed in the rotational mode as below:

$$
\begin{aligned}
& H P_{\text {Trans }}=\frac{s^{2}}{s^{2}+2 \xi \omega_{n} s+\omega_{n}{ }^{2}} \\
& L P_{\text {Trans }}=\frac{\omega_{n}{ }^{2}}{\mathrm{~s}^{2}+2 \xi \omega_{n} s+\omega_{n}{ }^{2}} \\
& H P_{\text {Rot }}=\frac{\mathrm{s}}{\mathrm{s}+\omega_{n}}
\end{aligned}
$$

where $\xi$ and $\omega_{n}$ are the damping ratio and cut-off frequency of the above filters.

The structure of the adaptive WF is similar to the classical WF [13] while it can vary the damping ratios and cut-off frequencies of the filters. According to Fig. 2, the inputs of the



Fig. 2. The schematic structure of the proposed adaptive WF with cogitation of the active and passive joints limitations.

classical and adaptive WFs for the longitudinal mode are linear acceleration and angular velocity signals shown as $\left(a_{x}\right)_{v e h}$ and $(\dot{\theta})_{v e h}$, respectively. It should be noted that the structure of lateral mode of the proposed adaptive WF is similar to the longitudinal mode while it has different inputs which are linear acceleration and angular velocity signals along $y$-axis shown as $\left(a_{y}\right)_{v e h}$ and $(\dot{\varphi})_{v e h}$. The heave and yaw channels consist of a translational channel and a rotational channel, respectively. The input signal of the heave mode is a linear acceleration signal along the $z$-axis $\left(a_{y}\right)_{v e h}$ (through the translational channel). The input signal of the yaw channel is the angular velocity signal along $z$-axis $(\dot{\psi})_{v e h}$ (through the rotational channel). The cut-off frequency of the adaptive WF is calculated using the fuzzy logic controller based on the accelerations, velocities and positions of the active joints, angular displacements of the passive upper joints, and the driving sensation error between the real vehicle driver and the MSP driver. In the following subsection, the human vestibular system and the structure of the proposed fuzzy logic controller are explained.

\section{A. Human Vestibular Perception Model}

The human vestibular model is located inside the inner ear of the human body as the main part of the human perceptual system, which is responsible for sensing the rotational and translational motions. The human vestibular system is composed of the otolith organ and semicircular canals. The otolith organ senses the translational motion signals and based on [35], the best transfer function model which relates the sensed specific force, $\hat{f}$, to the applied specific force, $f$, is as follows:

$\frac{\hat{f}}{f}=K_{\text {OTO }}\left(\frac{\left(\tau_{a} s+1\right)}{\left(\tau_{L} s+1\right)\left(\tau_{s} s+1\right)}\right)$

where $\tau_{a}, \tau_{L}$ and $\tau_{s}$ are respectively the neural processing term lead operator, the long time constant and the short time constant of the otolith organs. $\tau_{L}$ is $5.3(s), 5.33(s)$ and $5.33(s)$ in the $x$-, $y$ - and $z$-direction and $K_{\text {OTO }}, \tau_{s}$ and $\tau_{a}$ are $0.4,0.016(s)$ and $13.2(s)$ in all three translational directions.

Also, the semicircular canals are in charge of the rotational driving sensation. The transfer function model of the semicircular system based on [36], which relates the sensed angular velocity $\widehat{\omega}$ to the applied angular velocity $\omega$, is employed in this research as follows: 
$\frac{\widehat{\omega}}{\omega}=\left(\frac{\tau_{1} \tau_{a} s^{2}}{\left(\tau_{a} s+1\right)\left(\tau_{1} s+1\right)}\right)$

where $\tau_{a}$ and $\tau_{1}$ are the adaption time constant and the long time constant, respectively. $\tau_{a}=30(s)$ in all three rotational directions. It should be noted that $\tau_{1}=5.3(\mathrm{~s})$ for the pitch, $\tau_{1}=6.1(s)$ for the roll and $\tau_{1}=10.2(s)$ for the yaw.

The indicated models of the human vestibular system in Eq. (9) and Eq. (10) should be employed inside the proposed adaptive WF to reduce the motion sensation error with consideration of the dynamical and physical boundaries of the Hexapod MSP.

\section{B. Fuzzy Logic Control Unit}

Fig 3. a-c illustrates the structure of the fuzzy logic units for the adaptive WF to extract the appropriate cut-off frequency in the longitudinal mode. The same methodology can be followed to extract the model for the other modes including lateral, heave and yaw. According to Fig. 3.a the inputs of the fuzzy logic units for the high-pass filter of the translational channel are the maximum accelerations, velocities and positions of the leg between 6 legs shown as $\max \left(\ddot{l}_{i}\right), \max \left(\dot{l}_{i}\right)$ and $\max \left(l_{i}\right)$, respectively, as well as the error of the sensed specific force between the real vehicle driver and the MSP driver. The accelerations, velocities and positions of the legs are calculated based on Eq. (4), Eq. (5) and Eq. (6). Then, the maximum accelerations, velocities and positions of the legs are selected and normalised to be used as the inputs of the fuzzy control unit of the high-pass translational channel. The inputs are split into five different ranges known as very near, near, medium, far and very far. As the human vestibular system is unable to sense the exact amount of motion sensation, the motion sensation error for rotational and translational fuzzy blocks between the real vehicle and MSP driver can be categorised in five groups known as very negative, negative, zero, positive, and very positive. As the fuzzy unit cannot detect the direction of the Hexapod MSP in the next time step, the compensator unit is designed to enhance the ability of the fuzzy unit by detecting the directional Hexapod MSP's movement and modifying the cut-off frequency based on the current configuration of the Hexapod MSP. For instance, if the leg is close to its upper length limitation while it is shrinking, then the compensator unit decreases the generated cut-off frequency via the fuzzy unit to modify the current position of the Hexapod actuators. The outputs of the fuzzy units are the cut-off frequency of the WFs, and they are divided into five groups including very small, small, medium, big and very big. The higher cut-off frequency of the high-pass filter generates more restricted motions. The outputs of the fuzzy units should be multiplied by the scale factors based on the current position and workspace limitations of the Hexapod MSP. The mid-size Hexapod MSP leads to more restriction on the workspace area; therefore, a higher scale factor should be selected. Fig. 3.b shows the fuzzy unit that generates the cut-off frequency of the tilt coordination channel for the proposed adaptive WF. The first input of the fuzzy unit is the maximum angular displacement of the passive upper joints $\max \left(\beta_{i}\right)$. The primary cause for the rotational limitation of the Hexapod MSP is the limitation of its passive joints. The

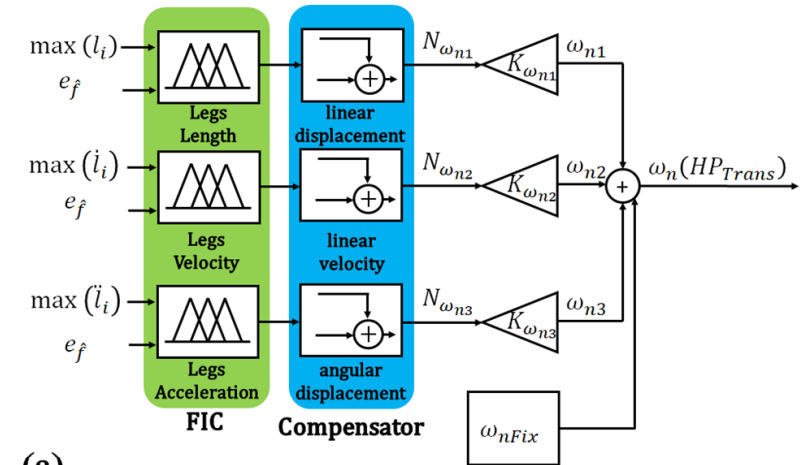

(a)

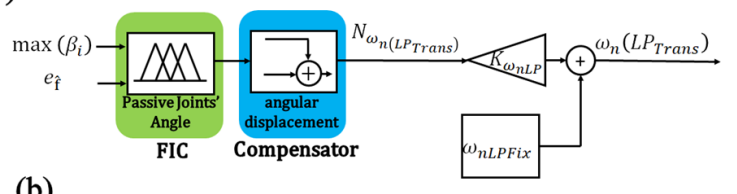

(b)

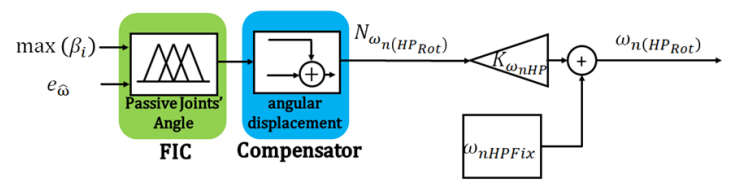

(c)

Fig. 3. The structure of fuzzy logic based adaptive WF for longitudinal channel (a): the high-pass filer of the translational mode;(b) the low-pass filer of the translational mode; (c) the high-pass filer of the rotational mode.

maximum usage of the Hexapod's rotational movement can be achieved by monitoring the angular displacement of the passive joints. The angular displacement is divided into five groups similar to the active joints' limitations known as very near, near, medium, far and very far. The second input is similar to the second input of the translational channel. However, the output of the rotational channel is quite the opposite of the translational channel's output. In fact, the higher cut-off frequency in lowpass filter generates less restricted angular motions. Fig. 3.c shows the fuzzy unit that produces the cut-off frequency for the rotational channel of the adaptive WF. The first input is the maximum angular displacement of the passive upper joints and the second input is the error of the sensed angular velocity between the real vehicle driver and the MSP driver. The rotational motion sensation error is categorised into five groups known as very negative, negative, zero, positive, and very positive. The scale factors are employed to increase the effectiveness of the fuzzy unit positively, and they have to be tuned based on the Hexapod MSP's boundaries.

\section{Membership Function and Rule}

Fig. 4.a presents the membership functions of the acceleration, velocity and position limitations of the active joints and the position limitations of the passive joints as the first inputs of the fuzzy logic units. Fig. 4.b-c shows the input membership functions for translational and rotational motion sensation error between the real vehicle driver and the MSP driver. The actual motion signals, including linear acceleration and angular velocity, were generated via the simulated vehicle user (using vehicle simulation environment soft body physics engine called Rigs of Rods (RoR) and developed MCA 




(a)

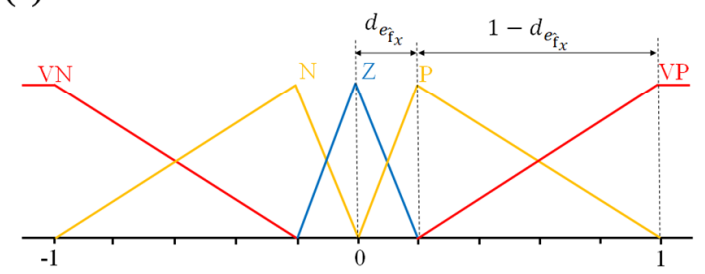

(b)



(c)

Fig. 4. The membership functions of (a): the input active joints' accelerations, velocities and positions, and passive joints' angular displacements; (b): the input sensed specific force's error; (c): the input sensed angular velocity's error.

connected to the motion platform in the simulation environment. This is employed inside Eq. (9) and Eq. (10) in order to calculate the actual translational and rotational motion sensations shown as $\left(\hat{f}_{x}\right)_{v e h}$ and $\left(\widehat{\omega}_{\theta}\right)_{v e h}$, respectively. Also, the translational and rotational motion sensation of the MSP's user are calculated using the extracted motion signals via the MCA inside Eq. (9) and Eq. (10) shown as $\left(\hat{f}_{x}\right)_{M S P}$ and $\left(\widehat{\omega}_{\theta}\right)_{M S P}$, respectively. The difference between these two values determines the translation and rotational motion sensation error for the MSP user. Between the trapezoidal, triangular, sigmoid, and gaussian membership function, the triangular membership function is chosen in this study because of the light computational burden, small data load and simple definition using its asymmetric form [28]. The inputs' membership functions, as shown in Fig. 4.a-c are overlapping. According to Fig. 4.c and d, there is a difference between the inputs' membership functions for the translational and rotational motion sensation error. It is due to the structure of the human otolith organs and semicircular canals [37]. The dead zone of the membership functions of the rotational sensation error is due to the human threshold unit as the MSP's driver is not supposed to sense the angular motion when there is only translational motion. Also, Fig. 5 presents the output membership functions of the fuzzy units to generate the cut-off frequency of the high- and low-pass filters. The outputs' membership functions, as shown in Fig. 5 are overlapping. Twelve parameters including $d_{\max \left(l_{i}\right)}, d_{\max \left(i_{i}\right)}, d_{\max \left(i_{i}\right)}$,

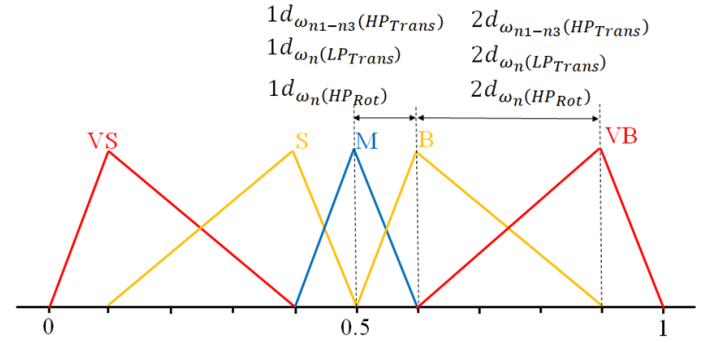

(b)

Fig. 5. The output generated cut-off frequency for high- and low-pass filters.

$d_{\max \left(\beta_{i}\right)}, 1 d_{\omega_{n 1-n 3}\left(H P_{\text {Trans }}\right)}, 2 d_{\omega_{n 1-n 3}\left(H_{\text {Trans }}\right)}, 1 d_{\omega_{n}\left(L P_{\text {Trans }}\right)}$, $2 d_{\omega_{n}\left(L P_{\text {Trans }}\right)}, 1 d_{\omega_{n}\left(H P_{R o t}\right)}, 2 d_{\omega_{n}\left(H P_{R o t}\right)}, d_{e_{\hat{\mathfrak{x}}_{x}}}$ and $d_{e_{\widehat{\omega}_{\theta}}}$ should be specified in order to determine the membership functions, as shown in Fig. 4.a-c and Fig. 5.

The multi-objective genetic algorithm (NSGA-II [38]) is used to extract the optimum value of these parameters using four different data-sets of white noise. The first sub-cost function is the translational and rotational motion sensation error between the real vehicle driver and the MSP driver. The second sub-cost function is specified based on the maximum acceleration, velocity and position of the legs, as well as the maximum angular displacement of the passive joints. The above-mentioned sub-cost function can be shown in integration form of the inputs and outputs as follows:

$J_{M C A 1}=w_{\hat{f}_{x}} \int\left(\left(\hat{f}_{x}\right)_{v e h}-\left(\hat{f}_{x}\right)_{M S P}\right) d t+w_{\widehat{\omega}_{\theta}} \int\left(\left(\widehat{\omega}_{\theta}\right)_{v e h}-\right.$

$\left.\left(\widehat{\omega}_{\theta}\right)_{M S P}\right) d t$

$J_{M C A 2}=w_{l} \int\left(\max \left(l_{i}\right)\right)^{2} d t+w_{i} \int\left(\max \left(i_{i}\right)\right)^{2} d t+$

$w_{i} \int\left(\max \left(\ddot{l}_{i}\right)\right)^{2} d t+w_{\beta} \int\left(\max \left(\beta_{i}\right)\right)^{2} d t$

where $w_{\hat{f}_{x}}, w_{\widehat{\omega}_{\theta}}, w_{l}, w_{i}, w_{i}$ and $w_{\beta}$ are the influence factors of the sensed specific force, sensed angular velocity, leg's position, leg's velocity, leg's acceleration and angular displacement of the passive upper joints. These factors can be determined by using both trial-and-error and expert knowledge of the system and its mechanisms. The adjustment factors of the multi-objective genetic algorithm are shown in Table II. These parameters have to be adequately defined to increase the precision and convergence speed of the method. In this study, a low mutation rate and high crossover fraction are chosen based on the work of De Jong and Michell $[39,40]$ which was later used by Asadi et al. [1, 18, 19] in MCA's domain and its effectiveness was proven. The high population size increases the computational burden of the model, while the low population size restricts the searchability of the genetic algorithm. In this study, the population size of 104 is selected to have a faster convergence time while still having a high search capability. The optimised values using the multiobjective genetic algorithm are shown in Table III. The same methodology can be followed for other channels including lateral, heave and yaw.

The Mamdani-type fuzzy is selected and the rules of the fuzzy logic controller are presented in Table IV for leg length of the translational channel. Also, Fig. 6 presents the surface of the translational fuzzy control rules for leg length fuzzy block. 
TABLE II

MUlti-OBJective TUNING PARAMETERS

\begin{tabular}{cc}
\hline \hline Parameters & Value \\
\hline & $d_{\max \left(l_{i}\right)} ; d_{\max \left(i_{i}\right)} ; d_{\max \left(\ddot{l}_{i}\right)} ; d_{\max \left(\beta_{i}\right)} ;$ \\
Variables & $1 d_{\omega_{n 1-n 3}\left(H P_{\text {Trans }}\right)} ; 2 d_{\omega_{n 1-n 3}\left(H P_{\text {Trans }}\right)} ;$ \\
& $1 d_{\omega_{n}\left(L P_{\text {Trans }}\right)} ; 2 d_{\omega_{n}\left(L P_{\text {Trans }}\right)} ; 1 d_{\omega_{n}\left(H P_{\text {Rot }}\right)} ;$ \\
& $2 d_{\omega_{n}\left(H P_{\text {Rot }}\right)} ; d_{e_{\mathfrak{f}_{x}}} ; d_{e_{\hat{\omega}_{\theta}}}$ \\
Maximum Generation & 45 \\
Population size & 104 \\
Crossover rate & 0.8 \\
Tolerance & $10^{-6}$ \\
Mutation function & Adaptive Feasible \\
\hline \hline
\end{tabular}

TABLE III

PARAMETERS OF THE MEMBERSHIP FUNCTIONS

\begin{tabular}{cc}
\hline \hline Parameters & Value \\
\hline$d_{\max \left(l_{i}\right)} ; d_{\max \left(i_{i}\right)} ; d_{\max \left(\ddot{l}_{i}\right)}$ & $0.2082 ; 0.4762 ; 0.2229$ \\
$d_{\max \left(\beta_{i}\right)} ; 1 d_{\omega_{n 1}\left(H P_{\text {Trans }}\right)} ; 1 d_{\omega_{n 2}\left(H P_{\text {Trans }}\right)}$ & $0.3752 ; 0.1211 ; 0.1505$ \\
$1 d_{\omega_{n 3}\left(H P_{\text {Trans }}\right)} ; 2 d_{\omega_{n 1}\left(H P_{\text {Trans }}\right)} ; 2 d_{\omega_{n 2}\left(H P_{\text {Trans }}\right)}$ & $0.3094 ; 0.1380 ; 0.0724$ \\
$2 d_{\omega_{n 3}\left(H P_{\text {Trans }}\right)} ; 1 d_{\omega_{n}\left(L P_{\text {Trans }}\right)} ; 2 d_{\omega_{n}\left(L P_{\text {Trans }}\right)}$ & $0.1196 ; 0.2139 ; 0.2090$ \\
$\left.1 d_{\omega_{n}\left(H P_{\text {Rot }}\right)} ; 2 d_{\omega_{n}\left(H P_{\text {Rot }}\right)}\right)$ & $0.2586 ; 0.2134$ \\
$d_{e_{\tilde{f}_{x}}} ; d_{e_{\widehat{\omega}_{\theta}}}$ & $0.2286 ; 0.2048$ \\
\hline \hline
\end{tabular}

TABLE IV

FuZzy Control Rules For LinEAR DisPlacement Weight PARAMETER TUNING

\begin{tabular}{|c|c|c|c|c|c|c|}
\hline \multirow{2}{*}{$W_{x}$} & & \multicolumn{5}{|c|}{$e_{\hat{f}_{x}}$} \\
\hline & & $\mathrm{VN}$ & $\mathrm{N}$ & Z & $\mathrm{P}$ & VP \\
\hline \multirow{4}{*}{$\frac{\max \left(l_{i}\right)}{\operatorname{limitation}\left\{l_{i}\right\}}$} & $\mathrm{VN}$ & VS & VS & VS & VS & VS \\
\hline & $\mathrm{N}$ & VS & $\mathrm{S}$ & S & $\mathrm{S}$ & VS \\
\hline & $\mathrm{M}$ & $\mathrm{S}$ & $\mathrm{M}$ & M & $\mathrm{M}$ & $\mathrm{S}$ \\
\hline & $\mathrm{F}$ & $\mathrm{M}$ & B & B & B & $\mathrm{M}$ \\
\hline & VF & B & VB & VB & VB & B \\
\hline
\end{tabular}

VN: very negative and very near; $\mathrm{N}$ : negative and near; $\mathrm{Z}$ : zero; $\mathrm{P}$ : positive; VP: very positive; M: middle and medium; F: far; VF: very far; VS: very small; S: small; B: big; VB: very big.

It should be noted that the rules for the legs' velocities and accelerations of the translational channel and passive joints' angles of the rotational channel are the same as listed in Table IV. Also, the rules for the passive joints' angle of tilt coordination channel are opposite to the translational and rotational channels. According to Table IV, the translational human sensation error and the position of the active joints are the inputs. The control actions are produced based on the rules of the fuzzy units. For instance, if the error of the sensed specific force between the real vehicle driver and the MSP driver is very positive, and the maximum length of the legs is close to its limitation, the cut-off frequency of the translational high-pass filter should be set to very big to penalise the motion of the Hexapod MSP and respect the boundaries of its active joints. The detected error of the sensed angular velocity or sensed specific force will affect the output when the legs are close to the neutral position to utilise the legs' position, velocity and acceleration limitations more efficiently.

\section{Results AND DisCussions}

\section{A. SimMechanic Modelling}

To validate the proposed adaptive fuzzy logic-based WF, the classical WF introduced by Conrad and Schmidt [13] and adaptive WF developed by Hwang et al. [24] and improved by

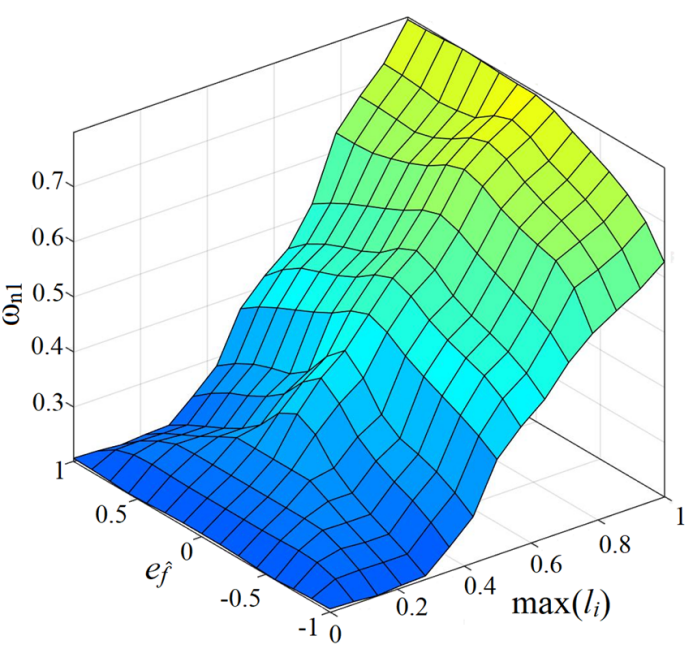

Fig. 6. The fuzzy rule surface of the leg length fuzzy block.

Asadi et al. [26] are designed and implemented using MATLAB software. Also, the inverse kinematics model of the Hexapod MSP is modelled to find the active joints' accelerations, velocities and positions as well as the angular positions of the passive joints. Fig. 7 shows the complete model of the proposed fuzzy logic-based adaptive WF, which is composed of the adaptive fuzzy MCA unit, human vestibular model and the inverse kinematics model. Also, the Hexapod MSP is developed using SimMechanics environment in MATLAB software to consider the dynamical and physical boundaries of the active and passive joints. The SimMechanic model is used to produce realistic results. The acceleration and angular velocity sensors on the moving platform of the Hexapod are used to monitor the actual sensed specific force and sensed angular velocity of the MSP. Therefore, it might not be possible to reproduce every vehicle motion signal using the MCA unit as the dynamical and physical limitations of the Hexapod MSP will be exceeded. The classical WF is designed based on [13]. The previous adaptive WF is designed based on consideration of the workspace limitations in the Cartesian space introduced by Asadi et al. [28]. The new fuzzy logicbased adaptive WF is designed and developed based on the proposed model in section III. The three methods are tuned to keep the MSP within its workspace limitations using the trialand-error method based on our expert knowledge of the system. The pre-scaling method is used to keep the end-effector inside the working area. Asadi et al. [41] proposed a nonlinear scaling method for optimal and model predictive WFs, respectively. Also, the same scale factors are selected for three methods to provide a fair comparison. The scale factors of the linear acceleration signal are $1 / 5,1 / 5$ and $1 / 3$ along the $x$-, $y$ - and $z$ axis, respectively. Also, the scale factor of the angular velocity signal is 1,1 and $1 / 2$ along roll-, pitch- and yaw-angle, respectively. Fig. 7 shows that the inverse kinematic model calculates the desired parameters for the adaptive WF to consider the active and passive joints' limitations in order to reproduce more accurate motion signals. It should be noted that the stability of the time-varying cut-off frequencies for the highand low-pass filters are studied numerically and practically $[25$, 


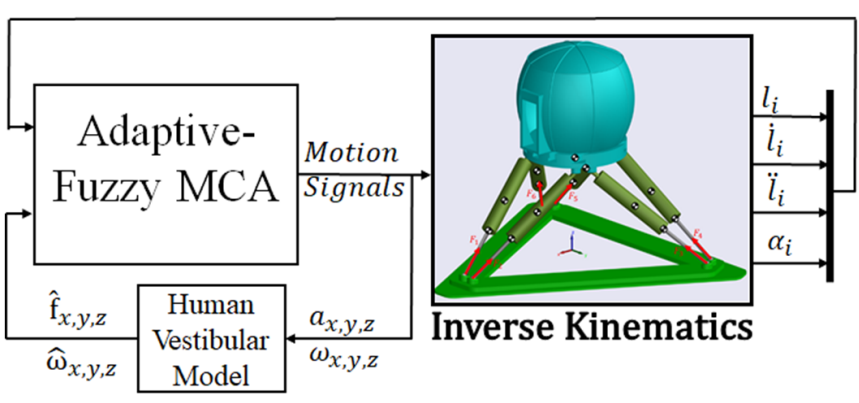

Fig. 7. The algorithm of the proposed adaptive WF.

26]. Initially, the different range of the cut-off frequencies are investigated to ensure that it will not make the system unstable. Then, the extracted period of the cut-off frequency is practically tested using the step and brake signal to guarantee the stability with the presence of sharp jumps in the filtered motion signal.

\section{B. Motion Scenario}

The motion scenario is recorded using a vehicle simulation environment soft body physics engine RoR. It includes sudden accelerations, turning the wheels, bumping, deceleration and braking during the 25 seconds of motions generated, as shown in Figs. 8. a-c. Fig. 8.a shows the road map including the start point and end point of motion. Also, Figs. 8.b-c shows the linear acceleration and angular velocity signals, respectively. The recorded motion signals are fed into classical, previous adaptive and proposed adaptive WFs, while they are connected to the motion platform in the simulation environment for assessing the MSP driver's sensation in order to show the efficiency of the proposed model.

Figs. 9. a-c show the sensed angular velocity for the real vehicle driver and Hexapod MSP driver using classical, previous adaptive and newly proposed adaptive WFs. The proposed fuzzy logic-based adaptive WF follows the shape of the actual sensed angular velocity signal better than classical and previous adaptive WFs. Also, the proposed fuzzy logicbased adaptive WF follows the shape of the real vehicle driver sensed angular velocity better when compared with the previous adaptive WFs along the roll-and pitch-angle. The correlation coefficient (CC) can be employed to compare the shape similarity of two signals, which is introduced by Asadi et al. [3]. The $\mathrm{CC}$ varies between 0 and 1 , and a higher value of $\mathrm{CC}$ means more similarity between the shape of two signals. The $\mathrm{CC}$ of the sensed angular velocity using proposed adaptive WF improved $12.28 \%, 6.33 \%$ and $6.54 \%$ along the roll-, pitchand yaw-angles, respectively when compared with the classical WF. Also, the newly proposed adaptive WF shows $2.5 \% 1$ and $5.88 \%$ improvement of the $\mathrm{CC}$ when compared with previous adaptive WF along the roll- and pitch-angles, respectively. The reduction of the $\mathrm{CC}$ along the yaw-angle using the proposed adaptive WF when compared with the previous adaptive WF is due to the consideration of the active joints' limitations including accelerations, velocities and positions. In other words, the previous adaptive WF uses the active and passive joints' limitations to generate more yaw-angle instead of rollor pitch-angle. Fig. 9. d-f show the sensed specific force for the



(a)
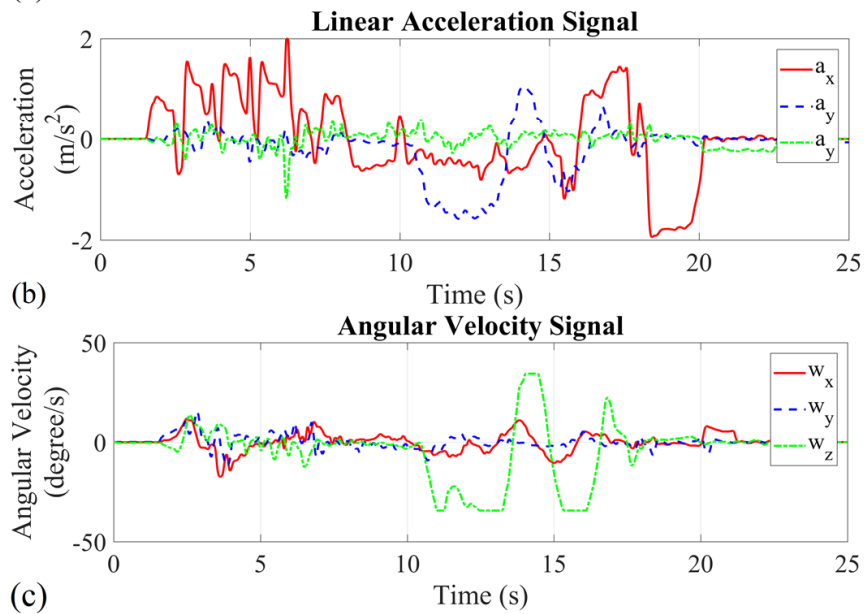

Fig. 8. The reference signal (real vehicle motions): (a) linear acceleration; (b) angular velocity.

real car and the Hexapod MSP user using the classical, previous adaptive and proposed adaptive WFs. The better shape similarity of the newly proposed adaptive WF is evident as the $\mathrm{CC}$ of the newly proposed adaptive WF is $6.63 \%, 2.88 \%$ and $5.08 \%$ better than the previous adaptive WF, and $15.90 \%$, $9.75 \%$ and $6.18 \%$ better than classical WF along $x$-, $y$ - and $z$ axis.

The root-means-square error (RMSE) determines the total reduction of the motion sensation error between the real vehicle driver and the MSP driver. The errors of sensed angular velocity between the real driver and the Hexapod MSP user using classical, previous adaptive and newly proposed adaptive WFs are shown and compared in Figs. 10. a-c. The RMSE of the sensed angular velocity using the proposed adaptive WF increased $43.47 \%, 16.80 \%$ and $5.10 \%$ along the roll-, pitch- and yaw-angles when compared with the classical WF. Also, the RMSE of the sensed angular velocity using the proposed adaptive WF increased and $18.11 \%$ and $1.98 \%$ along the rolland pitch-angle when compared with classical WF and previous adaptive WF. The higher RMSE of the sensed angular velocity along the yaw-angle using the proposed adaptive WF is due to the consideration of the active and passive joints limitations. Therefore, the algorithm decides to use the limitation along the roll- and pitch-angles, which are more essential in driving motion scenario. Also, Figs. 10. d-f show the reduction of the sensed specific force error using proposed adaptive WF along the $x$-, $y$ - and $z$-axis. The results of the human motion sensation in cooperation with the classical, previous and proposed adaptive WFs are summarised in Table V.

Figs. 11. a-c illustrate the linear motion of the Hexapod MSP 

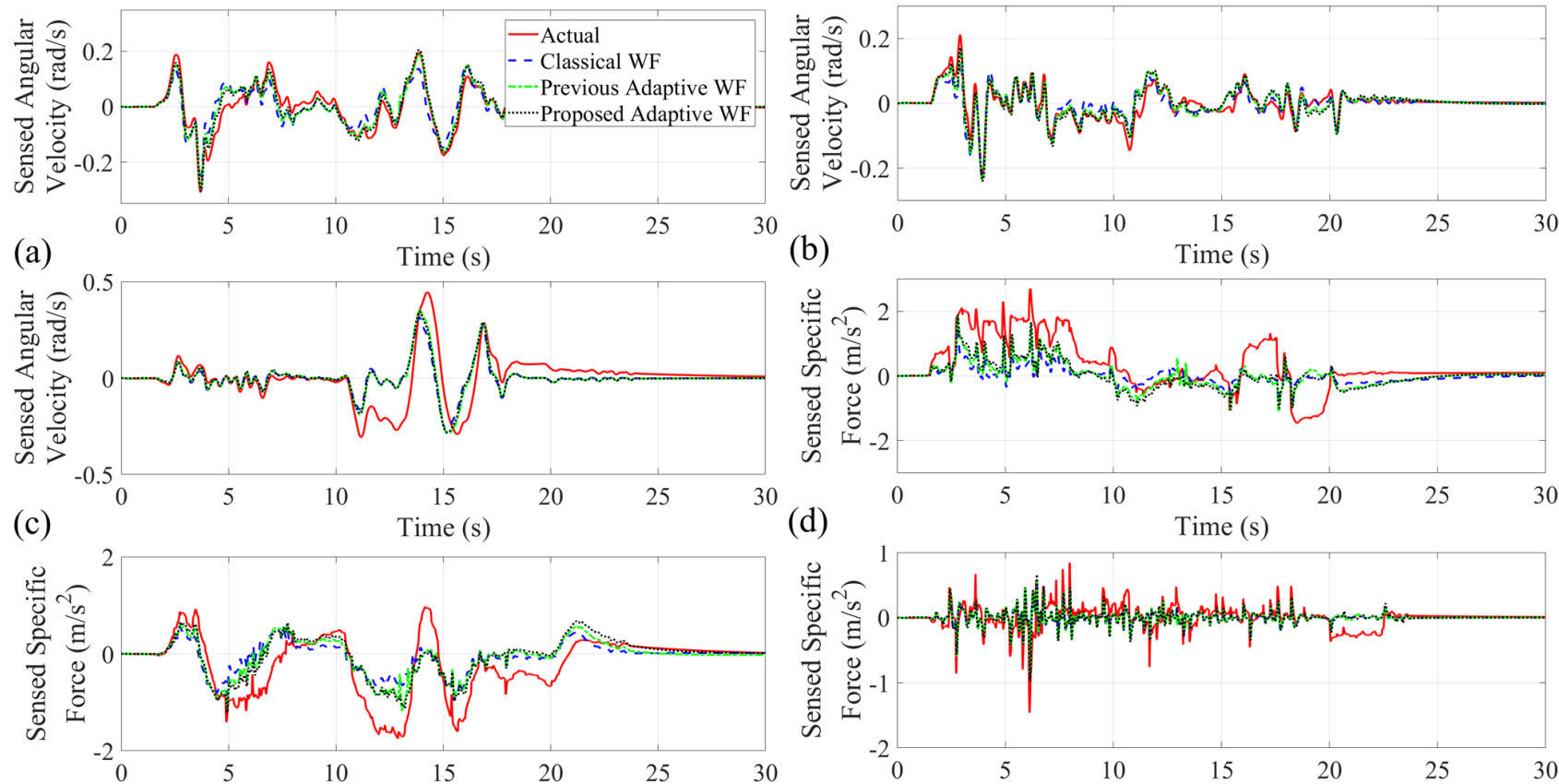

(e)

(b)

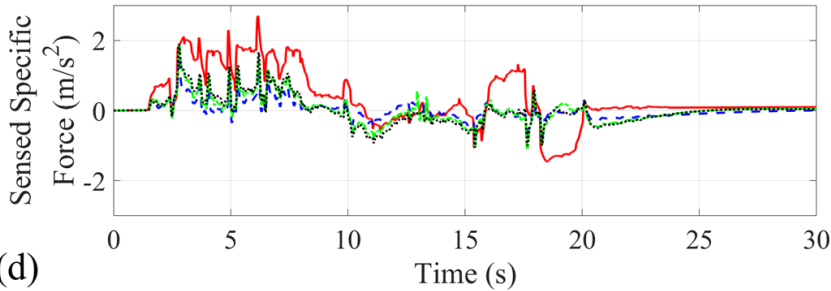

(d)



Fig. 9. The driving sensation for the actual, classical, pervious adaptive and proposed WFs along: (a) pitch-axis (b) roll-axis (c) yaw-axis (d) $x$-axis (e) $y$-axis (e) $z$-axis.
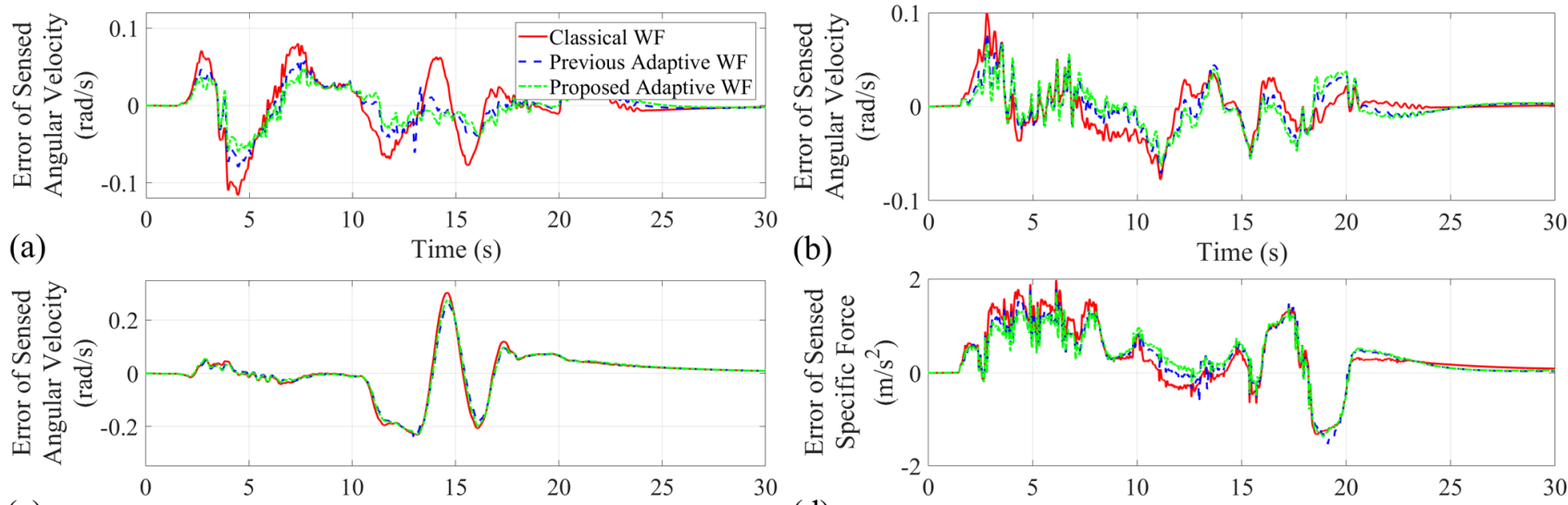

(c) Time (s)

(d)
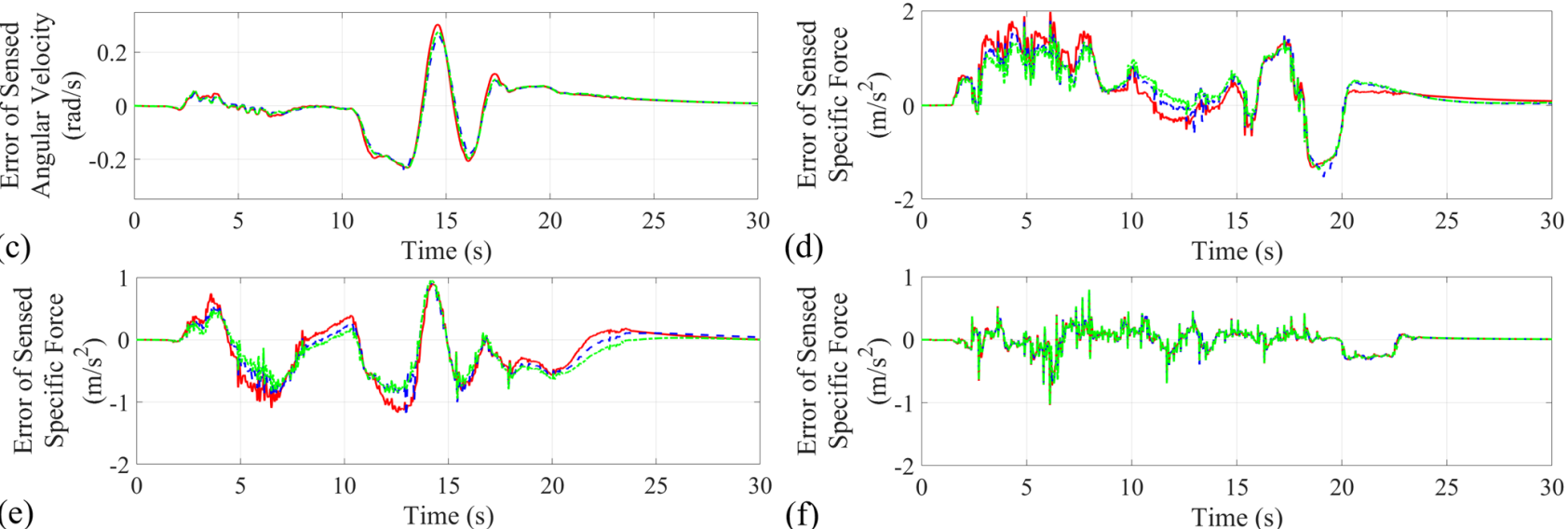

Fig. 10. The error of driving sensation for the classical, pervious adaptive and proposed WFs along: (a) pitch-axis (b) roll-axis (c) yaw-axis (d) $x$-axis (e) $y$-axis (e) $z$-axis.

using the classical, previous adaptive and proposed adaptive WFs. Also, Figs. 11. d-f illustrate the angular motion of the Hexapod MSP using the classical, previous adaptive and proposed adaptive WFs. The proposed adaptive WF considers the active and passive joints' limitations and can use the workspace area more wisely when compared with the classical and previous adaptive WFs. The proposed fuzzy logic-based adaptive WF can use the rotational motion of the end-effector better than the classical and previous adaptive WFs to reproduce more accurate low-frequency linear acceleration signals. Finally, Fig. 12 and Fig. 13 show the active joints' accelerations, velocities and positions as well as the passive joints' angular positions, respectively. Figs. 12. a-b show the Hexapod legs' lengths using the previous and proposed adaptive WFs. Also, Figs. 12. c-d show the velocity of the active joints using proposed and previous adaptive WFs. As the 

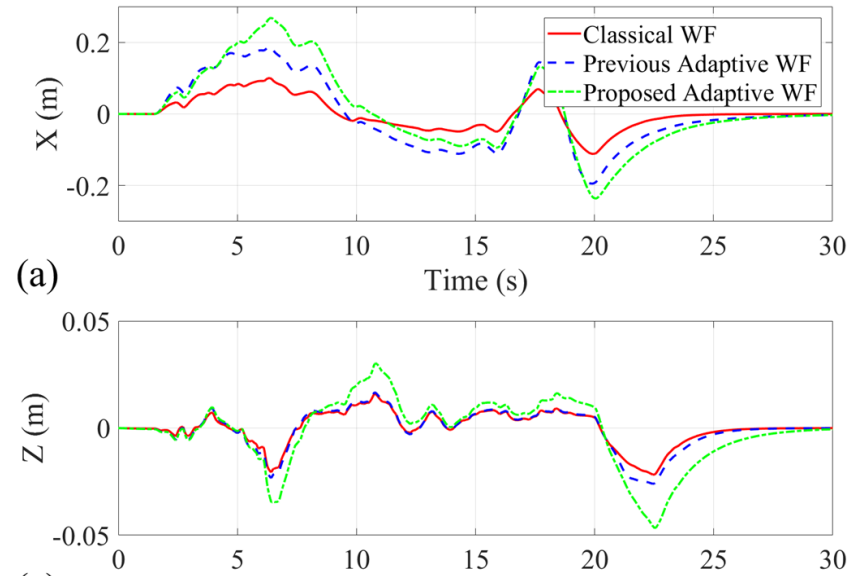

(c)

Time (s)


(d)

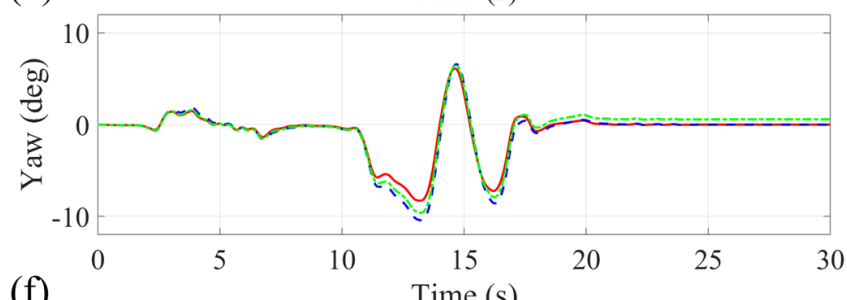

(e)

Time (s)

(f)

Time (s)

Fig. 11. Linear and angular motion of the Hexapod MSP using classical, previous adaptive and proposed adaptive WFs: (a) $x$-axis; (b) $y$-axis; (c) $z$-axis; (a) rollangle; (b) pitch-angle; (c) yaw-angle.

previous adaptive WF is not able to consider the lengths and velocities of legs, the controller unit of the Hexapod MSP saturates the accelerations, velocities and positions of the legs if legs hit the limitations. The leg 1 reaches the linear displacement limitation during 10-15 seconds of motion, as illustrated in Fig. 12.a. This causes the lower rotational motion sensation error along the yaw- angle using the previous adaptive WF. It means that the previous adaptive WF generates an unnecessary high yaw angle using unnecessary usage of the leg 1 length. On the other hand, the saturation of leg 1 length affects the quality of generated motion signal along other three modes. Figs. 12. e-f present the acceleration of the active legs using the proposed and previous adaptive WFs. It is evident that the proposed adaptive WF respects the dynamical and physical limitations of the actuators more than the previous adaptive WF. Figs. 13. a-c explain the angular motion of the passive joints during the motion scenario using the classical, previous adaptive and proposed adaptive WFs, respectively. The proposed adaptive WF uses the angular positions of the passive joints better than the classical and previous adaptive WFs. The results prove the efficiency of the proposed fuzzy logic- based adaptive WF when compared with the classical and previous adaptive WF. Using the proposed adaptive WF, the RMSE of motion sensation between the real vehicle driver and the MSP driver decreases in different modes compared to the classical and previous adaptive WFs. Also, the CC between the MSP and actual vehicle increases for all modes, which proves that the proposed method can generate accurate motion signals for the users. The proposed adaptive WF can be developed for different mechanisms, allowing them to use their dynamical and physical limitations to generate more accurate motion signals. Also, the proposed method can consider passive joints' positions to

TABLE V

The Result For the Classical, Previous Adaptive and Proposed Adaptive WFs

\begin{tabular}{cccc|ccc}
\hline \hline & & \multicolumn{3}{c}{ CC } \\
\cline { 2 - 7 } Index & Classical WF & $\begin{array}{c}\text { Previous } \\
\text { Adaptive WF }\end{array}$ & $\begin{array}{c}\text { Proposed } \\
\text { Adaptive WF }\end{array}$ & Classical WF & $\begin{array}{c}\text { Previous } \\
\text { Adaptive WF }\end{array}$ & $\begin{array}{c}\text { Proposed } \\
\text { Adaptive WF }\end{array}$ \\
\hline $\begin{array}{c}\text { Sensed Specific Force along } x \text { - } \\
\text { axis }\end{array}$ & 0.7074 & 0.6503 & 0.6247 & 0.6394 & 0.6950 & 0.7411 \\
$\begin{array}{c}\text { Sensed Specific Force along } y \text { - } \\
\text { axis }\end{array}$ & 0.4466 & 0.3960 & 0.3783 & 0.7645 & 0.8156 & 0.8391 \\
$\begin{array}{c}\text { Sensed Specific Force along } z \text { - } \\
\text { axis }\end{array}$ & 0.1546 & 0.1546 & 0.1526 & 0.4611 & 0.4659 & 0.4896 \\
$\begin{array}{c}\text { Sensed Angular Velocity along } \\
\text { pitch } \text {-angle }\end{array}$ & 0.0238 & 0.0202 & 0.0198 & 0.8538 & 0.8998 & 0.9079 \\
$\begin{array}{c}\text { Sensed Angular Velocity along } \\
\text { roll-angle }\end{array}$ & 0.0368 & 0.0254 & 0.0208 & 0.8500 & 0.9310 & 0.9544 \\
$\begin{array}{c}\text { Sensed Angular Velocity along } \\
\text { yaw-angle }\end{array}$ & 0.0881 & 0.0804 & 0.0836 & 0.6507 & 0.7209 & 0.6933 \\
\hline \hline
\end{tabular}

CC: Correlation Coefficient; RMSE: Root Mean Square Error; 




(a)

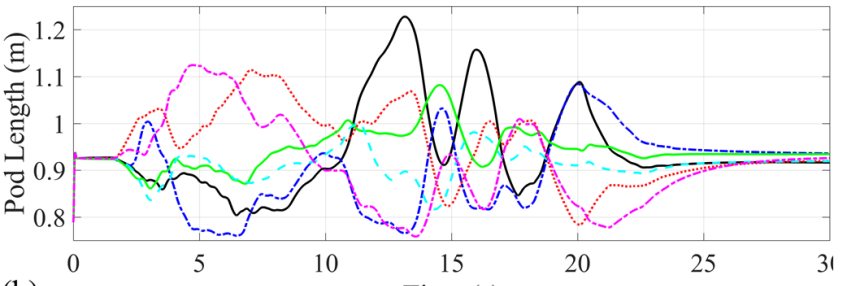

(b)

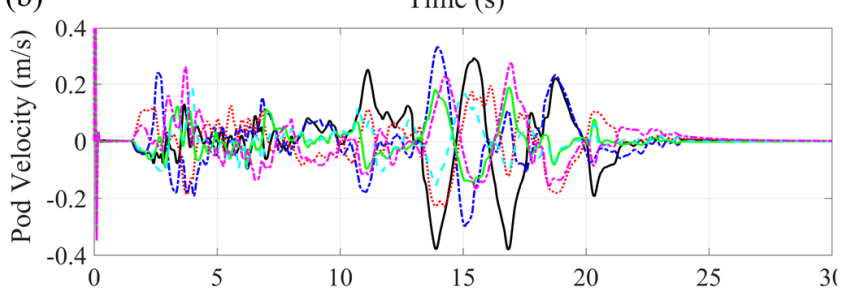

(c)

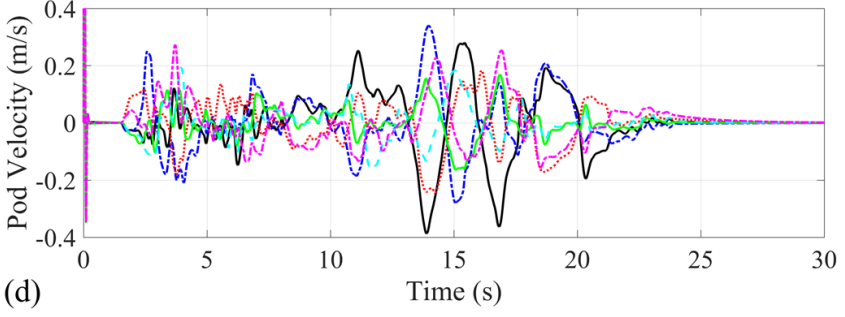

(d)

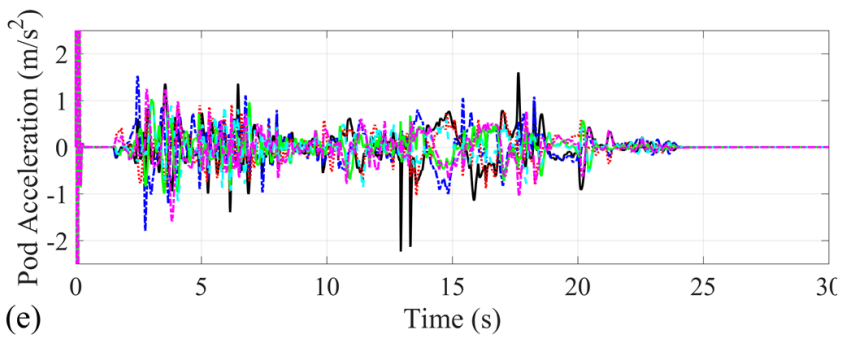

(e)



Fig. 12. (a) Legs' positions of the previous adaptive WF; (b) Legs' positions of the proposed adaptive WF; (c) Legs' velocities of the previous adaptive WF; (d) Legs' velocities of the proposed adaptive WF; (e) Legs' accelerations of the previous adaptive WF; (f) Legs' velocities of the proposed adaptive WF.

generate sustained linear acceleration motion signal.

\section{CONCLUSION}

A fuzzy logic-based adaptive WF is developed in this study for an Hexapod platform in order to consider the dynamical and

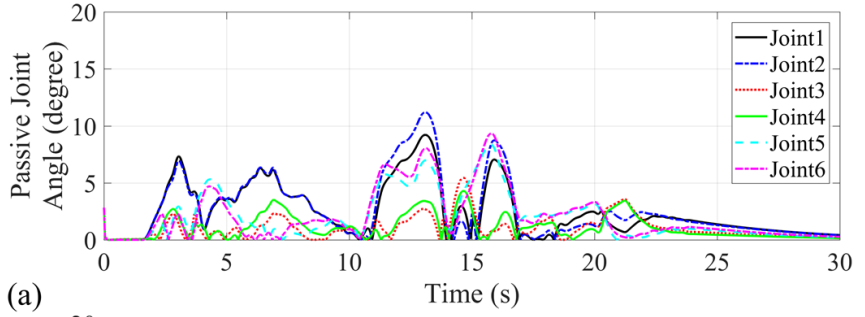

(a)



(b)

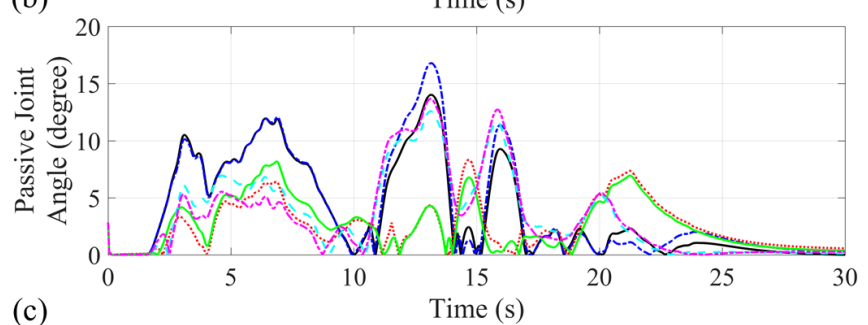

Fig. 13. Angular displacement of the upper passive joints (a): of the classical WF; (b): of the previous adaptive WF; (c) of the proposed adaptive WF.

physical boundaries of its actuators as well as the physical boundaries of its passive joints for reproducing the precise driving motion sensation for MSP drivers. As the classical WF cannot consider the physical limitations of the MSP in Cartesian space, it should be tuned based on the worst-case scenario to respect the workspace boundaries of the MSP. Also, the existing adaptive WFs [24, 26] only consider the physical boundaries of the MSP in Cartesian space, while the dynamical and physical boundaries of the active and passive joints are the main reason for the workspace limitations of the MSP. The consideration of the MSP in Cartesian space still can lead to poor usage of the available workspace, and it can lead to wrong motion cues.

This paper aims to improve the platform's workspace without any conservative motion using the fuzzy logic technique. The inverse kinematic model of the Hexapod MSP is introduced initially to be considered in developing the adaptive WF. Also, the fuzzy logic controllers are employed to generate suitable cut-off frequencies for the adaptive WFs. The fuzzy logic unit monitors the dynamical and physical limitations of the active and passive joints as well as the motion sensation error between the real vehicle driver and the MSP driver to generate suitable cut-off frequencies. The membership functions are tuned using a multi-objective genetic algorithm to reduce the motion sensation error and respect the active and passive joints' limitations. In addition, the compensator unit is designed to increase the efficiency of the proposed fuzzy logic controller by detecting the directional motion of the Hexapod MSP and adding a correction value to the fuzzy units' outputs. The proposed method is designed and implemented using 
MATLAB software and is compared with the classical and previous adaptive WFs. The better use of the workspace boundaries with better respect to the active and passive joints' limitations is achieved while decreasing the motion sensation error using the presented fuzzy logic-based adaptive WF. As a future work, a Kalman filter can be employed along with the proposed adaptive MCA as a supplementary filter to provide estimates for unknown variables, given the measurements observed over time, such as noise and simulator actuators' behaviors in high acceleration situations.

\section{APPENDIX}

The positions of the upper passive joints positions in reference to the local frame:

$$
\begin{aligned}
& { }_{\mathrm{P}} \mathbf{a}_{i}= \\
& {\left[\begin{array}{cccccc}
0.6428 & 0.6428 & 0.342 & -0.9848 & -0.9848 & 0.342 \\
-0.766 & 0.766 & 0.9397 & 0.1736 & -0.1736 & -0.9397 \\
0 & 0 & 0 & 0 & 0 & 0
\end{array}\right]}
\end{aligned}
$$

The positions of the lower passive joints positions in reference to world frame:

$\mathbf{b}_{i}=$

$$
\left[\begin{array}{ccc}
2.9971 & 2.9971 & -1.3852 \\
-0.1309 & 0.1309 & 2.6610 \\
0 & 0 & 0 \\
-1.6119 & -1.6119 & -1.3852 \\
2.5302 & -2.5302 & -2.6610
\end{array}\right]
$$

legs lengths variation:

$$
l_{i}=0.5-1.5 \quad i=1-6
$$

Mass of the end-effector, upper legs and lower legs:

$m_{p}=1216.9 ; m_{u}=143.9 ; m_{l}=92.1$

The end-effector, upper legs and lower legs inertia tensors in their local frames:

$$
\begin{aligned}
\mathbf{I}_{p} & =\left[\begin{array}{ccc}
304.5 & 0 & 0 \\
0 & 304.5 & 0 \\
0 & 0 & 304.5
\end{array}\right] \\
\mathbf{I}_{u} & =\left[\begin{array}{ccc}
67.2 & 0 & 0 \\
0 & 67.2 & 0 \\
0 & 0 & 67.2
\end{array}\right] \\
\mathbf{I}_{l} & =\left[\begin{array}{ccc}
43 & 0 & 0 \\
0 & 43 & 0 \\
0 & 0 & 43
\end{array}\right]
\end{aligned}
$$

\section{REFERENCES}

[1] H. Asadi, S. Mohamed, C. P. Lim, and S. Nahavandi, "Robus optimal motion cueing algorithm based on the linear quadratic regulator method and a genetic algorithm," IEEE Transactions on Systems, Man, and Cybernetics: Systems, vol. 47, no. 2, pp. 238 $254,2016$.

[2] H. Arioui, S. Hima, L. Nehaoua, R. J. Bertin, and S. Espié, "From design to experiments of a 2-DOF vehicle driving simulator," IEEE Transactions on Vehicular Technology, vol. 60, no. 2, pp. 357-368, 2010 .

[3] S. Pedrammehr, M. R. C. Qazani, H. Asadi, and S. Nahavandi, "Control System Development of a Hexarot-Based High-G Centrifugal Simulator," in ICIT, 2019, pp. 78-83.

[4] M. R. C. Qazani et al., "Kinematic analysis and workspace determination of hexarot-a novel 6-DOF parallel manipulator with a rotation-symmetric arm system," Robotica, vol. 33, no. 8, pp. $1686-1703,2015$.
[5] M. R. C. Qazani, H. Asadi, and S. Nahavandi, "High-Fidelity Hexarot Simulation-Based Motion Platform Using Fuzzy Incremental Controller and Model Predictive Control-Based Motion Cueing Algorithm," IEEE Systems Journal, 2019.

[6] M. R. C. Qazani, H. Asadi, S. Pedrammehr, and S. Nahavandi, "Performance analysis and dexterity monitoring of hexapod-based simulator," in 2018 4th International Conference on Control, Automation and Robotics (ICCAR), 2018: IEEE, pp. 226-231.

[7] H. Arioui, L. Nehaoua, S. Hima, N. Séguy, and S. Espié, "Mechatronics, design, and modeling of a motorcycle riding simulator," IEEE/ASME Transactions on Mechatronics, vol. 15, no. 5,pp. 805-818, 2010.

[8] R. J. Telban and F. M. Cardullo, "Motion cueing algorithm development: Human-centered linear and nonlinear approaches," NASA TechReport CR-2005-213747, 2005.

[9] H. Asadi, "Human perception-based washout filtering," Ph.D. dissertation, Deakin Univeristy, Victoria, Australia, 2015.

[10] H. Asadi, S. Mohamed, K. Nelson, and S. Nahavandi, "A linear quadratic optimal motion cueing algorithm based on human perception," in ACRA 2014: Proceedings of Australasian Conference on Robotics and Automation, 2014: Australian Robotics and Automation Association, pp. 1-9.

M. Dagdelen, G. Reymond, A. Kemeny, M. Bordier, and N. Maïki, "MPC based motion cueing algorithm: Development and application to the ULTIMATE driving simulator," in Conférence simulation de conduite, 2004, pp. 221-233.

[12] M. R. C. Qazani, H. Asadi, S. Khoo, and S. Nahavandi, "A Linear Time-Varying Model Predictive Control-Based Motion Cueing Algorithm for Hexapod Simulation-Based Motion Platform," IEEE Transactions on Systems, Man, and Cybernetic: Systems, 2019, doi: 10.1109/TSMC.2019.2958062.

[13] B. Conrad and S. F. Schmidt, "A study of techniques for calculating motion drive signals for flight simulators," 1971.

[14] P. R. MacNeilage, M. S. Banks, D. R. Berger, and H. H. Bülthoff, "A Bayesian model of the disambiguation of gravitoinertial force by visual cues," Experimental Brain Research, vol. 179, no. 2, pp. 263290, 2007.

[15] S. Casas, I. Coma, C. Portalés, and M. Fernández, "Towards a simulation-based tuning of motion cueing algorithms," Simulation Modelling Practice and Theory, vol. 67, pp. 137-154, 2016.

[16] J. Iskander et al., "From car sickness to autonomous car sickness: A review," Transportation research part F: traffic psychology and behaviour, vol. 62, pp. 716-726, 2019.

[17] R. V. Parrish, J. E. Dieudonne, and D. J. Martin Jr, "Coordinated adaptive washout for motion simulators," Journal of aircraft, vol. 12, no. 1, pp. 44-50, 1975

[18] L. Reid and M. Nahon, "Flight simulation motion-base drive algorithms: part 1. developing and testing equations," University of Toronto, 1985.

[19] L. Reid and M. A. Nahon, "Flight Simulation Motion-Base Drive Algorithms.: Part 2, Selecting The System Parameters," UTIAS report, no. N307, 1986.

[20] L. Nehaoua, H. Mohellebi, A. Amouri, H. Arioui, S. Espié, and A. Kheddar, "Design and control of a small-clearance driving simulator," IEEE Transactions on Vehicular Technology, vol. 57, no. 2, pp. 736-746, 2008.

[21] L. A. Zadeh, "Fuzzy sets," Information and control, vol. 8, no. 3, pp. 338-353, 1965.

[22] A. A. Khan, M. Abolhasan, W. Ni, J. Lipman, and A. Jamalipour, "A hybrid-fuzzy logic guided genetic algorithm (H-FLGA) approach for resource optimization in 5G VANETs," IEEE Transactions on Vehicular Technology, vol. 68, no. 7, pp. 69646974, 2019

[23] C. Zhang, Y. Shang, Z. Li, and N. Cui, "An interleaved equalization architecture with self-learning fuzzy logic control for seriesconnected battery strings," IEEE Transactions on Vehicular Technology, vol. 66, no. 12, pp. 10923-10934, 2017.

[24] T.-S. Hwang, S.-K. Yeh, J.-R. Lin, and W.-P. Su, "Adaptive motion washout filter design by using self-tuning fuzzy control," in 2009 IEEE/ASME International Conference on Advanced Intelligent Mechatronics, 2009: IEEE, pp. 811-815.

[25] H. Asadi, A. Mohammadi, S. Mohamed, and S. Nahavandi, "Adaptive translational cueing motion algorithm using fuzzy based tilt coordination," in International Conference on Neural Information Processing, 2014: Springer, pp. 474-482. 
[26] H. Asadi, A. Mohammadi, S. Mohamed, D. R. Zadeh, and S. Nahavandi, "Adaptive washout algorithm based fuzzy tuning for improving human perception," in International Conference on Neural Information Processing, 2014: Springer, pp. 483-492.

[27] H. Asadi, C. P. Lim, S. Mohamed, D. Nahavandi, and S. Nahavandi, "Increasing Motion Fidelity in Driving Simulators Using a FuzzyBased Washout Filter," IEEE Transactions on Intelligent Vehicles, vol. 4 , no. 2, pp. 298-308, 2019.

[28] H. Asadi, S. Mohamed, and S. Nahavandi, "Incorporating human perception with the motion washout filter using fuzzy logic control," IEEE/ASME Transactions on Mechatronics, vol. 20, no. 6, pp. 3276-3284, 2015.

[29] H. Asadi, S. Mohamed, K. Nelson, S. Nahavandi, and M. Oladazimi, "An optimal washout filter based on genetic algorithm compensators for improving simulator driver perception," in DSC 2015: Proceedings of the Driving Simulation Conference \& Exhibition, 2015: Max Planck Institute for the Advancement of Science, pp. 1-10.

[30] J.-P. Merlet, Parallel robots. Springer Science \& Business Media, 2006.

[31] C.-I. Huang, "Human visual-vestibular based (hvvb) adaptive washout filter design for vr-based motion simulator," in 2010 International Symposium on Computer, Communication, Control and Automation (3CA), 2010, vol. 2: IEEE, pp. 179-182.

[32] M. A. Nahon and L. D. Reid, "Simulator motion-drive algorithmsA designer's perspective," Journal of Guidance, Control, and Dynamics, vol. 13, no. 2, pp. 356-362, 1990.

[33] M. R. C. Qazani, S. Pedrammehr, and M. J. Nategh, "A study on motion of machine tools' hexapod table on freeform surfaces with circular interpolation," The International Journal of Advanced Manufacturing Technology, vol. 75, no. 9-12,pp. 1763-1771, 2014.

[34] M. R. C. Qazani, S. Pedrammehr, and M. J. Nategh, "An investigation on the motion error of machine tools' hexapod table," International Journal of Precision Engineering and Manufacturing, vol. 19, no. 4, pp. 463-471, 2018.

[35] H. Asadi, S. Mohamed, C. P. Lim, and S. Nahavandi, "A review on otolith models in human perception," Behavioural brain research, vol. 309, pp. 67-76, 2016.

[36] H. Asadi, S. Mohamed, C. P. Lim, S. Nahavandi, and E. Nalivaiko, "Semicircular canal modeling in human perception," Reviews in the Neurosciences, vol. 28, no. 5, pp. 537-549, 2017.

[37] J. A. Houck, R. J. Telban, and F. M. Cardullo, "Motion cueing algorithm development: Human-centered linear and nonlinear approaches," 2005.

[38] D. Kalyanmoy, Multi objective optimization using evolutionary algorithms. John Wiley and Sons, 2001.

[39] K. A. De Jong, "Analysis of the behavior of a class of genetic adaptive systems," 1975.

[40] M. Mitchell, An introduction to genetic algorithms. MIT press, 1998.

[41] H. Asadi, C. P. Lim, A. Mohammadi, S. Mohamed, S. Nahavandi, and L. Shanmugam, "A genetic algorithm-based nonlinear scaling method for optimal motion cueing algorithm in driving simulator," Proceedings of the Institution of Mechanical Engineers, Part I: Journal of Systems and Control Engineering, vol. 232, no. 8, pp. 1025-1038, 2018.

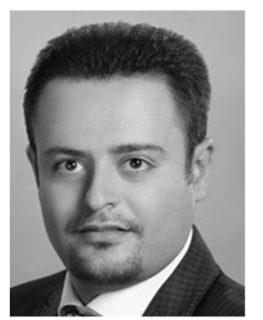

Mohammad Reza Chalak Qazani received the Bachelor of Engineering in manufacturing and production from University of Tabriz, Tabriz, Iran in 2010 and the master's degree in robotic and mechanical engineering from the Tarbiat Modares University, Tehran, Iran, in 2013.

$\mathrm{He}$ is currently a Ph.D. student in the Institute for Intelligent Systems Research and Innovation (IISRI), Deakin University. His current research interests include model predictive control, motion cueing algorithm and soft computing controllers.



Houshyar Asadi received the Bachelor of Engineering degree (First Class Hons.) in electricalcontrol systems in 2008, and Master degree in Industrial Electronic and Control Engineering from University of Malaya in 2012. He received the Ph.D. degree in human perception-based washout filtering using Artificial intelligence (AI) from the Institute for Intelligent Systems Research and Innovation (IISRI), Deakin University, Australia, in 2015.

He is currently a Senior Research Fellow of Control and Motion Analysis with the IISRI, Deakin University. His current research interests include computational intelligence, motion-based simulator technologies, motion cueing algorithms, robotics, control, and human factors in virtual environments.

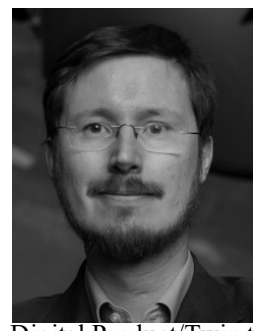

Tobias Bellmann is working as a scientist at the German Aerospace Center (DLR) since 2007. After finishing his $\mathrm{PhD}$ in the field robot path-planning, he became head of a workgroup with focus on the topics motion simulation and virtual reality, working on methods for modelling, simulating and visualizing mechatronic systems.

Since 2016 he heads the newly created DLR Systems and Control Innovation Lab (SCIL), commencing research on digitalization projects as the Digital Product/Twin together with various industry partners.



Shady Mohamed received the B.Sc. and M.Sc. degrees in information technology from Cairo University, Giza, Egypt, in 2000 and 2003, respectively, and the Ph.D. degree in control theory from Deakin University, Geelong, VIC, Australia, in 2009 . He is currently an associated professor with the Institute for Intelligent Systems Research and Innovation (IISRI), Deakin University, Australia.

$\mathrm{He}$ is interested in interdisciplinary research involving signal processing, control theory, human biodynamics, haptics and medical imaging.



Chee Peng Lim received his $\mathrm{PhD}$ degree from the University of Sheffield, UK, in 1997. His research interests include computational intelligence-based systems for pattern recognition, data mining, fault detection, condition monitoring, decision support, and process optimization. He has published over 450 technical papers in these areas.

$\mathrm{He}$ is currently a professor at Institute for Intelligent Systems Research and Innovation, Deakin University, Australia.

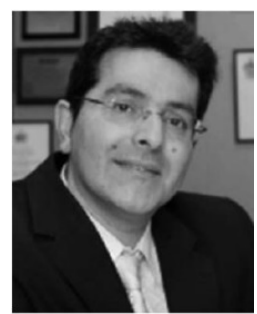

Saeid Nahavandi received a Ph.D. from Durham University, U.K. in 1991. He is an Alfred Deakin Professor, Pro Vice-Chancellor, Chair of Engineering, and the Founding Director of the Institute for Intelligent Systems Research and Innovation at Deakin University. His research interests include modeling of complex systems, robotics and haptics. He has published over 900 scientific papers in various international journals and conferences.

Professor Nahavandi is Editor-In-Chief: IEEE SMC Magazine, the Senior Associate Editor: IEEE Systems Journal, Associate Editor of IEEE Transactions on Systems, Man and Cybernetics: Systems, and IEEE Press Editorial Board member. Professor Nahavandi is a Fellow of IEEE (FIEEE), Engineers Australia (FIEAust), the Institution of Engineering and Technology (FIET). Saeid is a Fellow of the Australian Academy of Technology and Engineering (ATSE). 